\title{
Industrial Power Penalty Reduction by Engaging APFC Unit
}

\author{
Mamta Kokate ${ }^{1}$, Sheetal O. Bhoyar ${ }^{2}$, Sayali A. Sawarkar ${ }^{3}$ \\ Assistant Professor, Department of Electrical Engineering, RTMNU, Maharashtra, India ${ }^{1}$
}

B.E. Student, Department of Electrical Engineering, Shrimati Rajashree Mulak College of Engineering for Women,

Nagpur, Maharashtra, India ${ }^{2,3}$

\begin{abstract}
Power is the basic necessity for the economic development of country. Power consumption is increasing day by day at a very high rate. The modern system is so much dependent upon the use of electrical energy that it has become the part and parcel of our life. So we need to find out the causes of power loss and improve the power system. Due to industrialization the use of inductive load increases and hence power system losses its efficiency. So we need to improve the power factor with a suitable method. Now a days, the embedded is very much popular and most of the product are developed with microcontroller based embedded technology.
\end{abstract}

Keywords: Automatic power factor correction, microcontroller, embedded technology, capacitor bank.

\section{INTRODUCTION}

The industrialization is primarily increasing. In the industrial sector the various motoring loads are continuously running and generating the inductive load. So the power factor in this system gets reduced due to the inductive reactive power. But the electricity board has a standard limits regarding the power factor values and if the power factor goes below the specified limit; the electricity company charges the penalty to the industrial consumers. The Inductive loads affect the power factor so the power system losses its efficiency. In the present trend the designs are moving also towards the miniature architecture; this can be achieved in a product by using programmable device. Whenever we are thinking about any programmable devices then the embedded technology comes into forefront. The embedded is now a day very much popular and most of the product are developed with Microcontroller based embedded technology.

The Automatic Power factor Correction device is a very useful device for improving efficient transmission of active power. Automatic power factor correction device reads power factor from line voltage and line current by determining the delay in the arrival of the current signal with respect to voltage signal from the function generator with high accuracy by using an internal timer. This time values are then calibrated as phase angle and corresponding power factor. Then the values are displayed in the LCD modules. Then the motherboard calculates the compensation requirement and accordingly switches on different capacitor banks. This is developed by using 8051 microcontroller. Automatic power factor correction techniques can be applied to the industries, power systems and also households to make them stable and due to that the system becomes stable and efficiency of the system as well as the apparatus increases.

Recently, the technical revolution made embedded technology cheaper, so that it can be applied to all the fields. The pioneer manufactures of Power system and protection system such as SIMENS, LARSON \& TUBRO, and CUTLER HAMPER etc. manufacturing power factor improvement devices on embedded technology.

1. Power Factor Theory:

A) Types of power:

Active Power: The actual amount of power being used, or dissipated, in a circuit is called the true power. It is measured in watts and is symbolized mathematically by the capital letter P. Active power is a function of circuit's dissipative element, such as resistances( $R)$.

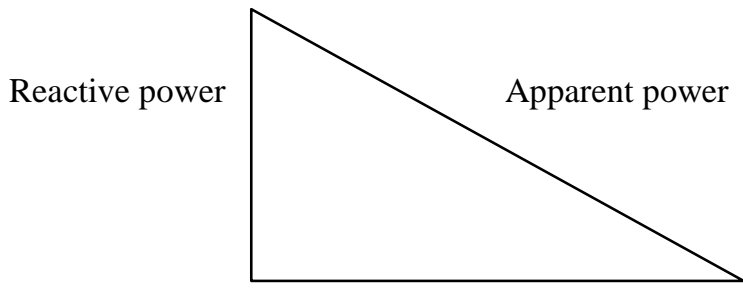

Active Power

Figure 1: Power Triangle

Reactive Power: Reactive loads such as inductors and capacitors dissipate zero power, but the fact that they drop voltage and draw current gives the perception that they do dissipate power. This "dissipated power" is called the reactive power and is measured in Volt-Amps-Reactive (VAR) and is symbolized by the capital letter Q, and it is the function of circuit's reactance (X).

Apparent Power: The combination of the active and reactive power is called apparent power. It is the product of a circuit's voltage and current, without reference to phase angle. Apparent power is measured in Volt-Amps (VA) and is symbolized by the capital letter S. Apparent power is a function of a circuit's total impedance $(\mathrm{Z})$

B) Power factor:

Power factor is the ratio between the active power $(\mathrm{kW})$ to the total apparent power (kVA) consumed by an a.c. electrical equipment or a complete electrical installation. 


$$
\text { Power Factor }(P F) \frac{\text { Active power }}{\text { Apparent power }}
$$

It is a measure of how efficiently electrical power is converted into useful work output. The ideal power factor is unity, or one. Anything less than one means that extra power is required to achieve the actual task at hand.

A poor power factor can be the result of either a significant phase difference between the voltage and current at the load terminals, or it can be due to a high harmonic content or distorted current waveform. A poor power factor due to an inductive load can be improved by the addition of power factor correction. Power factor is the relation between current and voltage. So power factor is also defined as the cosine of the phase difference between current and voltage. It can be mathematically expressed as $\cos \varnothing$, where $\varnothing$ is the phase difference between current and voltage.

Basically AC power circuits, have resistive loads (like heaters) or inductive loads (like motors) or capacitive loads (like power supplies). Depending upon the loads the current phasor can be in-phase with voltage, lagging the voltage or leading the voltage.

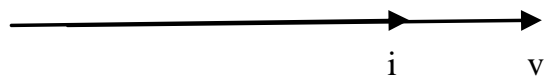

Figure 2: Phasor diagram for purely resistive load

For purely resistive load current is in phase with the voltage. Therefore phase difference $\varnothing=0^{\circ}$. Thus giving power factor, $\cos \emptyset=1$. Hence the power factor for purely resistive load is unity.

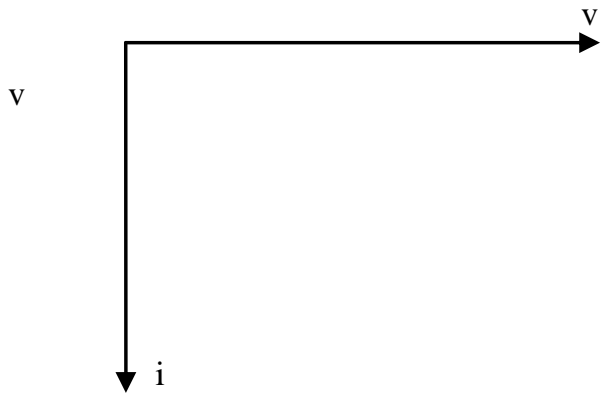

Figure 3: Phasor diagram for purely inductive load.

In a purely inductive circuit, the current lags the voltage. Therefore the phase difference between current and voltage $\varnothing=90^{\circ}$. Hence power factor is lagging.

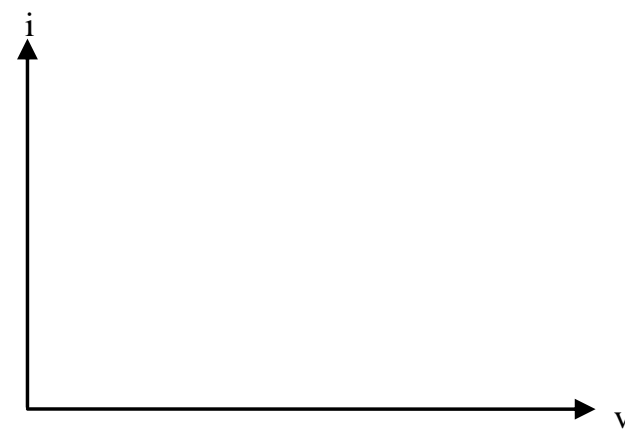

Figure 4: Phasor diagram for purely capacitive load.
In a purely capacitive circuit, the current leads the voltage therefore the phase difference between current and voltage $\varnothing=90^{\circ}$.thus the power factor is said to be leading.

C) Causes of low power factor:

Low Power factor is caused by inductive loads. Inductive loads require the current to create a magnetic field that produces the desired work. The result is an increase in reactive and apparent power and a decrease in the power factor, or efficiency, of a system. Since the power factor is defined as the ratio of $\mathrm{KW}$ to $\mathrm{KVA}$, we see that low power factor results when $\mathrm{KW}$ is small in relation to KVA. An inductive load includes transformers, induction motors, and induction generators, high intensity discharge lighting. These inductive loads constitute your distribution system. This increase in reactive power results in large angle between KW and KVA. This large angle decreases the power factor. The efficiency of inductive equipment and system power factor will vary depending on its manufacturer, design, size and age. Most inductive equipment has a nameplate with operating data, including its power factor at rated load.

D) Power factor correction:

Capacitive power factor correction is applied to circuits which include induction motors as a means of reducing the inductive component of the current and thereby reduce the losses in the supply. There should be no effect on the operation of the motor itself. An induction motor draws current from the supply that is made up of resistive components and inductive components. The current due to the leakage reactance is dependent on the total current drawn by the motor, but the magnetizing current is independent of the load on the motor. The magnetizing current will typically be between $20 \%$ and $60 \%$ of the rated full load current of the motor. The magnetizing current is the current that establishes the flux in the iron and is very necessary if the motor is going to operate. The magnetizing current does not actually contribute to the actual work output of the motor. It is a catalyst that allows the motor to work properly. The magnetizing current and the leakage reactance can be considered as the passenger components of current that will not affect that the power drawn by the motor, but contribute to the power dissipated in the supply and distribution system.

E) Disadvantages of low power factor:

i) Large Line Losses (Copper Losses):

Line Losses is directly proportional to the square of current ' $\mathrm{I}^{2}$ ' therefore, larger the current, the greater the line losses.

ii) Large kVA rating and size of electrical equipments:

Power factor is inversely proportional to the $\mathrm{kVA}$ i.e. $\operatorname{Cos} \Phi=\mathrm{kW} / \mathrm{kVA}$

Therefore, lower the power factor, the larger the $\mathrm{kVA}$ rating of machines also, the larger the $\mathrm{kVA}$ rating of machines, the larger the size of machines, the larger the cost of machines.

iii) Greater conductor size and cost:

In case of low power factor, current will be increased, thus to transmit this high current, we need the larger size of conductor. Also, the cost of large size of conductor will be increased. 
iv) Poor voltage regulation and large voltage drop:

$$
\text { Voltage drop }=\mathrm{V}=\mathrm{IZ} \text {. }
$$

With low power factor, current will be increased. So the larger the current, the larger the voltage drop. In case of low power factor there would be large voltage drop which cause low voltage regulation.

v) Low efficiency:

In case of low power factor, there would be large voltage drop and large line losses and this will cause the system or equipments efficiency too low. Due to low power factor, there would be large line losses; therefore, alternator needs high excitation, thus, generation efficiency would be low.

vi) Penalty from electric power supply

Electrical power Supply Company imposes a penalty of power factor below 0.95 lagging in electric power bill. So industries must improve power factor above 0.95 .

\section{PRINCIPLE}

Automatic power factor correction device is developed based on a microcontroller IC 8051. The voltage and current sample is converted into a square wave using a zero cross detector. The voltage and current sample signals are feed to the microcontroller at INT0 and INT1 and the difference between the arrivals of waveform indicate the phase angle difference.

The difference is measured with high accuracy by using internal timer. This time value is calibrated and the phase angle and the corresponding power factor is also calculated. This value is displayed in the LCD modules after converting suitably displayed in the LCD modules after converting suitably. The capacitor banks are switched as per the calibration in steps.

\section{METHODOLOGY}

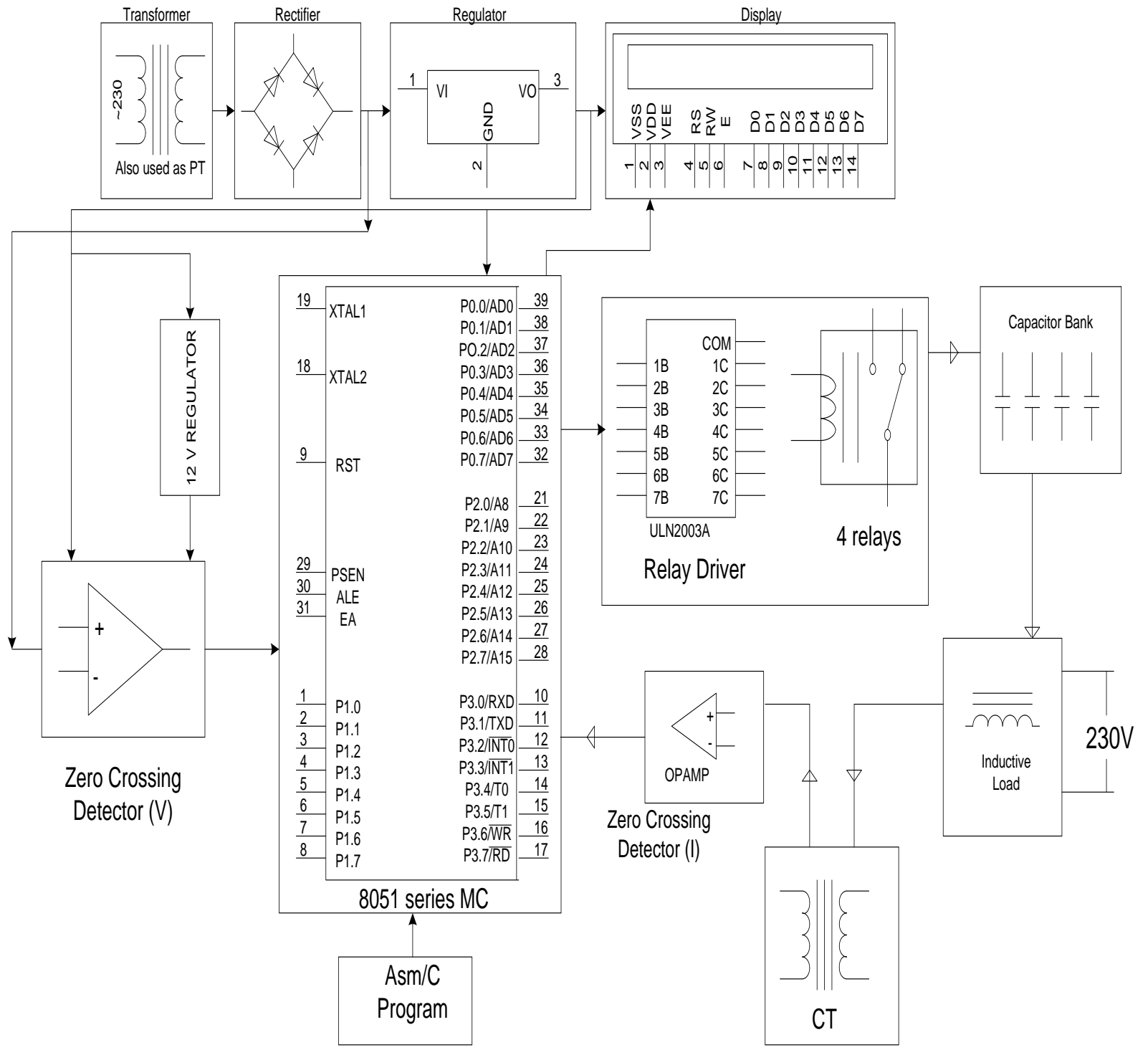

Figure 5: Block Diagram 
i) Power supply:

In power supply we are using step-down transformer. The $230 \mathrm{~V}$ ac input supply is given to the primary of the transformer. Transformer is an electromechanical static device which transforms power from one circuit to another without changing its frequency. Due to the magnetic effect of the coil the flux induced in the primary is transferred to the secondary coil.

ii) Rectifier:

The main function of the rectifier is to convert the ac voltage to the dc output. The diode is a primary component in most of the rectifier circuits since it conducts in one direction. This property of diode convert the sinusoidal voltages with zero average value into waveforms that contains both ac and dc components (pulsating dc). It is a full wave bridge rectifier.

iii) Voltage Regulator:

The main function of the voltage regulator is to convert the variable output DC voltage into the constant DC voltage which is required for the supply for the microcontroller and zero crossing detector.

iv) Zero Crossing Detectors:

The zero crossing detector circuit is an important application of the op-amp comparator circuit. It can also be called as sine to square wave convertor. It is used to detect sine wave zero crossing from positive half cycle to negative half cycle. The reference voltage with which the input voltage is to be compared, must be made zero (Vref $=0$ ).

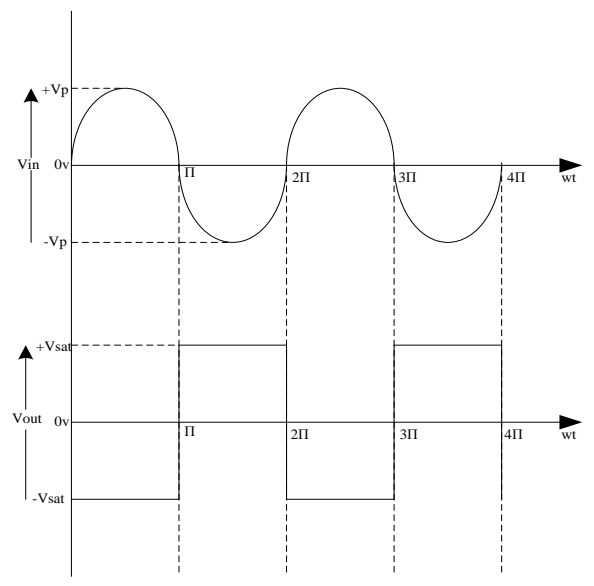

Figure 6: Waveforms of ZCD

The input sine wave is given as an Vin. To measure the time difference between two waves we need to detect zero crossing of two waves. Zero crossing detector produces an output state change whenever the input crosses the reference input. In this case the reference input connected to ground. The zero crossing detectors are shown in fig.

The output is driven into $-\mathrm{v}_{\text {sat }}$ when the input signal passes through zero to positive direction, conversely, when input signal passes through zero to negative direction the output switches to positive $\mathrm{v}_{\text {sat }}$.the waveforms for ZCD is shown in figure.

5) Microcontroller:

Microcontroller is a IC chip that executes programs for controlling other devices or machines. It is a micro (small size as an integrated circuit chip) device which is used for control of other devices and machines, that's why it is called microcontroller. It is a microprocessor having RAM, ROM and I/O ports. 8051 microcontroller is used in automatic power factor correction panel. The microcontroller receives the load current in the line and gives the signal to the relay driver and simultaneously connects the capacitors as per the requirement.

6) Relay:

A relay is an electrically operated switch. Many relays use an electromagnet to operate a switching mechanism mechanically, but other operating principles are also used. Relays are used where it is necessary to control a circuit by a low-power signal or where several circuits must be controlled by one signal. Current flowing through the coil of the relay creates a magnetic field which attracts a lever and changes the switch contacts. The coil current can be on or off so relays have two switch positions and most have double throw switch.

6) Relay Driver:

Relay Driver is interfaced with the microcontroller output. It is used to drives the multiple relays as per the compensation required. Relay driver IC

7) LCD:

LCD stands for liquid crystal display. It is a flat panel display or other electronic visual display that uses the light modulating properties of liquid crystals. LCD is used to show the present power factor. $16 \times 2$ LCD is connected with 8051 microcontroller. It is available in a 16 pin package with back light, contrast adjustment function and each dot matrix has $5 \times 8$ dot resolution.

8) Capacitor Bank:

Capacitors can be included for compensation of power factor through relay. A capacitor bank is a group of several capacitors of the same rating that are connected in series or parallel with each other to store electrical energy. The resulting bank is then used to counteract or correct a power factor lag or phase shift in an ac power capacitor does. They are designed to store electrical energy The most common use of a capacitor bank for ac power supply error correction is in industrial environments which use a large number of transformers and electric motors. Since this equipment uses an inductive load, they are susceptible to phase shifts and power factor lags in the power supply which can result in a loss of system efficiency if left uncorrected. By incorporating a capacitor bank in the system, the power lag can be corrected at the cheapest cost for the company when compared to making significant changes to the company power grid or system that is supplying the equipment.

\section{REQUIREMENTS}

1) Hardware Requirements: Microcontroller 8051 IC LM339

LCD

Relays

Relay driver IC

Switches

Resistors

Capacitors 
Switches

Resistors

Capacitors

Diodes

Transformers

Voltage regulator

2) Software Requirements:

i) Embedded C-language:

Embedded $\mathrm{C}$ is a set of language extension for the $\mathrm{C}$ programming language by the $\mathrm{C}$ standards committee to address commonality issues that exists between $\mathrm{C}$ extensions for different embedded systems. Embedded $\mathrm{C}$ programming requires non-standards extensions to the $\mathrm{C}$ language in order to support exotic features such as fixed point arithmetic, multiple distinct memory banks and basic input/output operations. Programming of 8051 is done using embedded $\mathrm{C}$ and then converted to Hex code to be dump into microcontroller IC.

ii) Compiler:

Compilers are programs used to convert a High Level Language to object code. Desktop compilers produce an output object code for the underlying microprocessor, but not for other microprocessors. The programs written in one of the HLL like ' $\mathrm{C}$ ' will compile the code to run on the system for a particular processor like 8051. For example compilers for Dos platform is different from the Compilers for Unix platform so if one wants to define a compiler then compiler is a program that translates source code into object code. The compiler derives its name from the way it works, looking at the entire piece of source code and collecting and reorganizing the instruction. See there is a bit little difference between compiler and an interpreter. Interpreter just interprets whole program at a time while compiler analyses and execute each line of source code in succession, without looking at the entire program. The advantage of interpreters is that they can execute a program immediately. Secondly programs produced by compilers run much faster than the same programs executed by an interpreter. However compilers require some time before an executable program emerges. Now as compilers translate source code into object code, which is unique for each type of computer, many compilers are available for the same language.

\section{EXPECTED RESULTS}

1) Advantages of improved power factor:

2) Better utilization of electrical machines

3) Better utilization of electrical lines

4) Reduction of losses

5) Decreased monthly energy cost

6) Efficient electrical system

7) Overloading is avoided

8) Efficiency of supply system and apparatus increases

\section{CONCLUSION}

It can be concluded that power factor correction technique can be applied to the industries, power systems and also house hold to make them stable and due to that the system becomes stable and efficiency of the systems as well as the apparatus increases. When the detected power absorbed by the load is greater than the compensator rating, the power factor will not be corrected to unity but certainly, will be improved and the apparent power supplied by the AC supply will be reduced. They achieve better power quality by reducing the apparent power drawn from the AC supply and minimizing the power transmission losses. Hence the efficiency of the systems as well as the apparatus increases.

\section{REFERENCES}

[1] Sapna Khanchi \& Vijay Kumar Garg, "Power Factor Improvement of Induction Motor by using Capacitors", International Journal of Engineering Trends \& Technology (IJETT), Volume 4, issued 7July 2013.

[2] Jain Sandesh, Thakur Shivendra Singh and Phulambrikar S.P., "Improve Power Factor And Reduce the Harmonic Distortion of the System", International Journal of Advanced Research in Computer Science and Software Engineeriong. Volume 1(5), issued November 2012.

[3] JBV Subrahmanyam, S.Radha Krishna Reddy, P.K. Sahoo, N.Madhukar Reddy, C.Sashidhar, "A Novel Method for Improvement of Power Factor in Wind Mill Power station" International Journal of Engineering Technology and Advanced Engineering", Volume 2, issue 2 February 2012.

[4] Abhinav Sharma, Shavet Sharma, Parveen Lehana \& Saleem Khan, "To Analysis the Effect of Combination Load on the Power Factor" , International Journal of Advanced Research in Computer Science and Software Engineering, volume 3, issue 8, August 2013.

[5] Anant Kumar Tiwari, "Automatic Power Factor Correction Using Capacitive Bank", International Journal of Engineering Research and Applications, Volume 4, issued February 2014. 